Research Article

\title{
Action Strategy Analysis in Probabilistic Preference Movement-Based Three-Way Decision
}

\author{
Chunmao Jiang (iD) and Shubao Zhao (i) \\ School of Computer Science and Information Engineering, Harbin Normal University, Harbin 150025, \\ Heilongjiang Province, China \\ Correspondence should be addressed to Shubao Zhao; machinelearner@126.com
}

Received 22 July 2020; Revised 3 November 2020; Accepted 6 November 2020; Published 9 December 2020

Academic Editor: Zeshui Xu

Copyright (c) 2020 Chunmao Jiang and Shubao Zhao. This is an open access article distributed under the Creative Commons Attribution License, which permits unrestricted use, distribution, and reproduction in any medium, provided the original work is properly cited.

\begin{abstract}
The trisecting-acting-outcome model is a methodology of "thinking in threes," which is the main idea of the three-way decision (3WD). It consists of three components: trisecting, acting, and outcome evaluation. A strategy selection method in a movementbased three-way decision (M-3WD) has been proposed in previous work. However, conflicting information widely existing in the information system has not yet been given sufficient consideration. The conflicting information brings massive noisy strategies when mining action strategies in three regions. This paper proposed a novel three-way decision model for action strategy set, which can analyze and classify strategies by introducing credibility and coverage. The model can remove noisy strategies and choose strategies more suitable for the need of decision makers. To evaluate and select an optimal action strategy, we analyze the probabilistic preference in a movement-based three-way decision. The approach determines the probability of movement by using the evidence theory (D-S) theory. The optimal action strategy is selected by analyzing the difference between the ideal movement and the actual movement, the lower the difference, the better the strategy. We give an example of medical decision-making to illustrate the effectiveness of the proposed method.
\end{abstract}

\section{Introduction}

Three-way decision [1] is an effective way of solving complex problems, whose core idea can be formulated as a three-step process within trisecting-acting-outcome (TAO) [2]. The trisecting is to divide a whole into three regions that are disjoint or weakly joint. The acting is to devise action strategies to process objects in the three regions. The outcome evaluation is to evaluate the effectiveness of the action strategy. The movement-based three-way decision [3] aims to mine actionable rules in three regions and transfer objects from the unfavorable region to the favorable region. We use an example of medical-making to illustrate the main ideas of the TAO model. In medical decision-making, one typically divides a set of suspected patients into three groups: suspected patients who have the disease, suspected patients who do not have the disease, and suspected patients who still need a further examination. According to the diagnosis result, the doctor may take some actions to turn the suspected patients who have the disease into disease-free, check the suspected patients who still need a further examination whether having the disease, and retain the suspected patients who do not have the disease. At last, the doctor develops new treatment protocols by evaluating the effects of treatment and medical examination.

Since the three-way decision was putting forward, it has expanded from a special three-way decision to a general three-way decision [4]. There are many studies on theories, such as three-way classification $[5,6]$, three-way clustering [7-12], three-way concept analysis [13, 14], three-way conflict analysis $[15,16]$, three-way game theory $[17,18]$, three-way support systems [19], three-way granular computing $[20,21]$, and three-way multiple attribute decision $[22,23]$. Yao $[2,24]$ systematically studied the main ideas of three-way decision and the granular computing model based on three-way decision. Qi and Wei [25] combined formal 
concept analysis with three-way decision and put forward three-way concept analysis theory. Yu [26] introduced threeway decision into clustering analysis and proposed threeway clustering approach. Wang and Yao [27] applied contraction and expansion in mathematical morphology to the three-way decision and proposed a three-way clustering algorithm based on mathematical morphology. In terms of application, three-way decision shows its unique superiority in image recognition [28, 29], mail filtering [30], medical decision-making [19], stream computing [31], recommendation system [32], and cloud computing [33].

Researchers have long focused on constructing a trisection from a whole $[5,6,34]$. Research on acting and outcome evaluation is still in its infancy. Gao and Yao [3] proposed a movement-based three-way decision model by mining actionable rules in three regions. Jiang and Yao $[35,36]$ proposed three-way decision models for quantity movement and probability movement. The corresponding outcome evaluation methods are also proposed.

In real life, the database usually stores a lot of inconsistent information [37], which is caused by missing values, duplicate, and errors. It is difficult to avoid even after many rounds of data processing. Inconsistent information refers to the data in an information system, whose condition attributes are the same but derive different decision results. In a movement-based three-way decision, the decision maker usually has a particular preference for different regions. The preference relationship will prompt the decision maker to construct action strategies in three regions. Objects will be moved from the unfavorable region to the favorable region according to the preference of decision-makers. The existence of conflicting information makes it inevitable to mine many inconsistent rules. The action strategy generated by inconsistent rules may induce multiple different decision results so that the movement-based three-way decision usually exhibits the characteristics of probability movement [35]. Determining the probability of movement and selecting an optimal action strategy are urgent problems that need to be solved.

This paper constructs a three-way decision model for strategy set by introducing the concepts of credibility and coverage [38]. The model can remove noisy strategies in the strategy set. To evaluate and select an optimal action strategy, we analyze the probabilistic preference in a movement-based three-way decision. The D-S theory determines the probability of movement. The optimal action strategy can be selected by analyzing the difference between the ideal movement and the actual movement, the lower the difference, the better the strategy.

The remainder of this paper is organized as follows. Section 2 reviews the TAO model of three-way decision and introduces the approach of constructing an action strategy. Section 3 analyses the uncertainty of the action strategy through an example of medical decision-making. Then, we propose a novel three-way decision model for the action strategy set. Section 4 analyses the probabilistic preference in M-3WD. The D-S theory is used to determine the probability of movement. The optimal action strategy is selected by analyzing the difference between the ideal movement and the actual movement. Section 5 illustrates the effectiveness of the proposed method with an example of medical decisionmaking. Section 6 gives a summary and planning for future work.

\section{Preliminaries}

In this section, we review the TAO model of three-way decision and the movement-based three-way decision.

2.1. TAO of Three-Way Decision. "Thinking in threes" $[2,39]$ is a kind of granular computing thought consistent with human cognition. After the summary and refinement by Yao $[1,24,40]$, the three-way decision theory is formed. The TAO model of the three-way decision is given in Figure 1. The trisecting function, denoted by solid lines with arrows, is to divide a whole into three pairwise disjoint or weakly joint regions $P_{1}, P_{2}$, and $P_{3}$. The function, denoted by dashed lines, is mining actionable rules in three regions. The function of acting, denoted by dashed lines with arrows, is to devise action strategies for three regions. The three regions before acting are represented by $P_{1}, P_{2}$, and $P_{3}$. The new three regions after acting are represented by $P_{1}^{\prime}, P_{2}^{\prime}$, and $P_{3}^{\prime}$. The outcome evaluation is to measure the effect of the trisection and action strategy.

The most fundamental issue of the three-way decision is how to construct a trisection from a whole with reasonable interpretations. Researchers have constructed three regions based on rough sets [40-43], fuzzy sets [34], shadowed sets $[44,45]$, intuitionistic fuzzy sets [46, 47], vague sets [48] and soft sets [49]. A three-way decision model with an ordered relationship is defined as follows.

Definition 1. Suppose that OB is a finite nonempty set of objects. $E: \mathrm{OB} \longrightarrow(L,<)$ is an evaluation function on set OB. For $x \in \mathrm{OB}, E(x)$ is an evaluation function value of $x$. Given a pair of thresholds $(\alpha, \beta) \in V \times V$ with $\beta \leq \alpha$, we trisect $\mathrm{OB}$ into three pairwise disjoint regions:

$$
\begin{aligned}
& P_{1}=\{x \in \mathrm{OB} \mid E(x) \geq \alpha\}, \\
& P_{2}=\{x \in \mathrm{OB} \mid \beta<E(x)<\alpha\}, \\
& P_{3}=\{x \in \mathrm{OB} \mid E(x) \leq \beta\} .
\end{aligned}
$$

The three regions satisfy the following two conditions:

(1) $P_{1} \cup P_{2} \cup P_{3}=\mathrm{OB}$

(2) $P_{1} \cap P_{2}=\Phi, P_{1} \cap P_{3}=\Phi, P_{2} \cap P_{3}=\Phi$

The $P_{1}$ region consists of objects with evaluation function value $E(x)$ greater than or equal to $\alpha$. The $P_{3}$ region consists of objects with evaluation function value $E(X)$ less than or equal to $\beta$. The $P_{2}$ region consists of objects with evaluation function value $E(x)$ between the two thresholds.

The acting aims to design an appropriate action strategy to process three regions, according to trisection. An efficient action strategy can make decision maker increase benefits or reduce costs. The decision maker hopes to adopt an appropriate action strategy to move the object from unfavorable to the favorable region. 


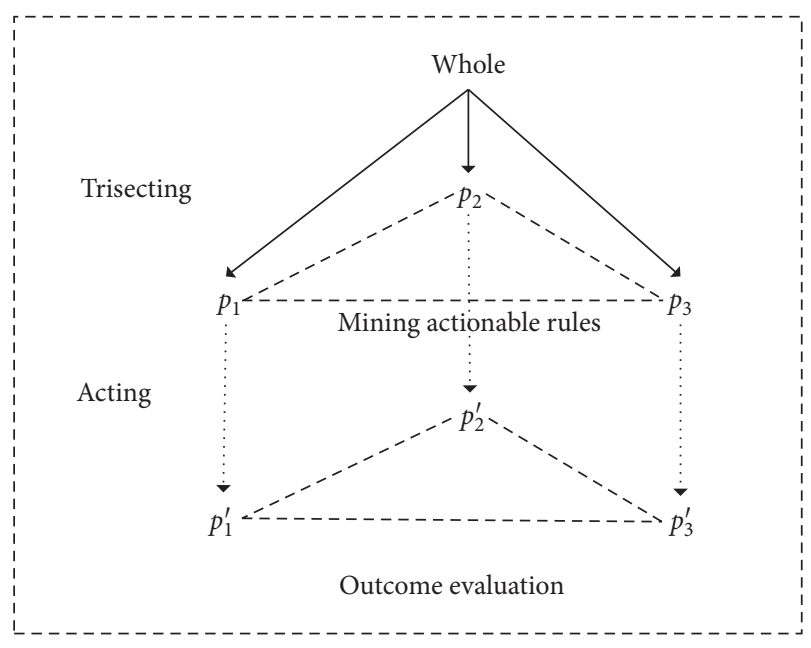

Figure 1: TAO model of trisecting-acting-outcome.

The outcome evaluation is to evaluate the effect of trisecting and acting. We can construct an evaluation function to evaluate the quality of trisecting:

$$
Q\left(\pi_{(\alpha, \beta)}\right)=w_{P_{1}} Q\left(P_{1}\right)+w_{P_{2}} Q\left(P_{2}\right)+w_{P_{3}} Q\left(P_{3}\right),
$$

where $Q\left(\pi_{(\alpha, \beta)}\right)$ denotes the quality of trisection by a pair of threshold $(\alpha, \beta), Q\left(P_{i}\right), i=1,2,3$, denotes the qualities or utilities of each regions, and $w_{P_{i}}, i=1,2,3$, denotes the weights of each regions. For acting, we can construct an evaluation function to evaluate the effect of action strategy:

$$
Q\left(\pi^{\prime} \mid \pi\right)=Q\left(\pi^{\prime}\right)-Q(\pi)
$$

where $Q(\pi)$ and $Q\left(\pi^{\prime}\right)$ denote the qualities or utilities of original three regions and new three regions.

2.2. Movement-Based Three-Way Decision. Gao and Yao [3] proposed a movement-based three-way decision by introducing actionable rules into the three-way decision. The actionable rules first proposed by Ras [50, 51]. It means that a user can mine actionable rules and moving objects to generate benefits. The movement-based three-way decision aims to mine action strategy in three regions and move objects from unfavorable regions to the favorable region.

Definition 2 (see [3]). Suppose that $[x]$ and $[y]$ are equivalence classes in different regions. We can get two decision rules:

$$
\begin{aligned}
& r_{[x]}:\left[\wedge_{s \in A_{s}} s=f_{s}(x)\right] \wedge\left[\wedge_{f \in A_{f}} f=f_{f}(x)\right] \Rightarrow d=f_{d}(x), \\
& r_{[y]}:\left[\wedge_{s \in A_{s}} s=f_{s}(y)\right] \wedge\left[\hat{f \in A}_{f} f=f_{f}(y)\right] \Rightarrow d=f_{d}(y),
\end{aligned}
$$

where $r_{[\cdot]}, \in\{x, y\}$, is decision rule, $A_{s}$ is a set of stable attributes, $f_{s}(\cdot)$ is the value of attribute $s, A_{f}$ is a set of flexible attributes, $f_{f}(\cdot)$ is the value of attribute $f$, and $f_{d}(\cdot)$ is the value of decision attribute $d$.
Definition 3 (see [3]). Suppose that $[x]$ and $[y]$ are equivalence classes in different regions, where $[x]$ is the equivalence class that needs to be moved and $[y]$ is the target equivalence class. If a user wants to move the object from one region to another, we can design an appropriate action strategy, that is,

$$
\begin{array}{r}
r_{[x]} \rightsquigarrow r_{[y]}:\left[{\hat{f \in A_{f}}}_{f} f_{f}(x) \rightsquigarrow f_{f}(y)\right] \Rightarrow f_{d}(x) \rightsquigarrow f_{d}(y), \\
\text { subject to }\left[{\hat{s \in A_{s}}}_{s} f_{s}(x)=f_{s}(y)\right],
\end{array}
$$

where $r_{[x]^{-} \rightarrow r_{[y]}}$ is actionable rules from $[x]$ to $[y]$, $\wedge_{s \in A_{s}} f_{s}(x)=f_{s}(y)$ means that $[x]$ and $[y]$ have the same value of stable attributes, and $\wedge_{f \in A_{f}} f_{f}(x) \rightsquigarrow f_{f}(y)$ means that the value of flexible attributes $f$ is changed from $f_{f}(x)$ to $f_{f}(y)$.

\section{Three-Way Decision for Action Strategy Set}

This section illustrates conflicting information through an example of medical decision-making and constructs the corresponding three-way decision model.

3.1. A Motivational Example. In real life, an information system often has much inconsistent information. The inconsistent information leads to a large number of inconsistent rules when mining actionable rules. It is not helpful to the decision maker and may even mislead them into choosing the wrong action strategy. The following example of medical decision-making illustrates conflicting information and the impact on the movement-based three-way decision.

Table 1 is a medical-decision information table. There are 20 suspected patients and six symptoms or attributes. All condition attributes had been discretized. The values of some attributes are grouped and reassigned as follows. Age is categorized into three groups, i.e., 0-20, 20-60, and 60+; they are reassigned to value 1 to 3 , respectively. Sex is categorized as female and male; they are reassigned to value 1 and 0 . Cholesterol is categorized into three groups, i.e., $0-199,200-239$, and 240+; they are reassigned to value 1 to 3 , respectively. Blood pressure is categorized into three groups, i.e., 0-89, 90-139, and 140+; they are reassigned to value 1 to 3 , respectively. Blood sugar is categorized into three groups, i.e., $0-3.9,4.0-7.8$, and 7.9+; they are reassigned to value 1 to 3 , respectively. Among them, cholesterol, blood pressure, and blood sugar are abbreviated as chol, pb, and bs. Age and sex are stable attributes, and others are flexible attributes. The symbol "+" stands for suspected patients who have the disease. The symbol "-" stands for suspected patients who have not the disease. The symbol "?" stands for suspected patients who need a further examination.

All objects are divided into following 8 equivalence classes based on their condition attributes: 
TABle 1: A decision table for medicine.

\begin{tabular}{|c|c|c|c|c|c|c|}
\hline & Age & Sex & Cholesterol & Blood pressure & Blood sugar & Result \\
\hline$x_{1}$ & 3 & 1 & 2 & 1 & 1 & + \\
\hline$x_{2}$ & 3 & 1 & 2 & 1 & 1 & + \\
\hline$x_{3}$ & 2 & 1 & 3 & 2 & 2 & - \\
\hline$x_{4}$ & 3 & 0 & 2 & 3 & 2 & $?$ \\
\hline$x_{5}$ & 3 & 0 & 2 & 3 & 2 & - \\
\hline$x_{6}$ & 2 & 1 & 3 & 2 & 2 & - \\
\hline$x_{7}$ & 3 & 0 & 2 & 2 & 2 & - \\
\hline$x_{8}$ & 3 & 0 & 1 & 2 & 3 & + \\
\hline$x_{9}$ & 3 & 0 & 2 & 3 & 2 & - \\
\hline$x_{10}$ & 2 & 0 & 3 & 3 & 3 & $?$ \\
\hline$x_{11}$ & 3 & 0 & 2 & 2 & 2 & - \\
\hline$x_{12}$ & 3 & 0 & 1 & 2 & 2 & - \\
\hline$x_{13}$ & 3 & 0 & 1 & 2 & 2 & - \\
\hline$x_{14}$ & 3 & 0 & 1 & 1 & 1 & + \\
\hline$x_{15}$ & 3 & 0 & 1 & 1 & 1 & + \\
\hline$x_{16}$ & 3 & 0 & 2 & 3 & 2 & + \\
\hline$x_{17}$ & 3 & 0 & 2 & 3 & 2 & - \\
\hline$x_{18}$ & 2 & 1 & 3 & 2 & 2 & - \\
\hline$x_{19}$ & 3 & 0 & 1 & 2 & 3 & - \\
\hline$x_{20}$ & 2 & 0 & 3 & 3 & 3 & + \\
\hline
\end{tabular}

$$
\begin{aligned}
& {[x]_{1}=\left\{x_{1}, x_{2}\right\},} \\
& {[x]_{2}=\left\{x_{3}, x_{6}, x_{18}\right\},} \\
& {[x]_{3}=\left\{x_{4}, x_{5}, x_{9}, x_{16}, x_{17}\right\},} \\
& {[x]_{4}=\left\{x_{7}, x_{11}\right\},} \\
& {[x]_{5}=\left\{x_{8}, x_{19}\right\},} \\
& {[x]_{6}=\left\{x_{10}, x_{20}\right\},} \\
& {[x]_{7}=\left\{x_{12}, x_{13}\right\},} \\
& {[x]_{8}=\left\{x_{14}, x_{15}\right\} .}
\end{aligned}
$$

Doctors divide suspected patients into three regions $P_{1}$, $P_{2}$, and $P_{3}$ based on the diagnosis result:

$$
\begin{aligned}
& P_{1}=\left\{x_{3}, x_{5}, x_{6}, x_{7}, x_{9}, x_{11}, x_{12}, x_{13}, x_{17}, x_{18}, x_{19}\right\}, \\
& P_{2}=\left\{x_{1}, x_{2}, x_{8}, x_{14}, x_{15}, x_{16}, x_{20}\right\}, \\
& P_{3}=\left\{x_{4}, x_{10}\right\} .
\end{aligned}
$$

Taking $[x]_{3}$ as an example, according to equation (4), we can construct the decision rules, that is,

$$
r_{[x]_{3}}: \text { age }=3 \wedge \operatorname{sex}=0 \wedge \text { chol }=2 \wedge \text { bp }=3 \wedge \text { bs }=3 \Rightarrow \text { result }= \begin{cases}+, & =1, \\ -, & =3, \\ ?, & =1 .\end{cases}
$$

There are five different objects in $[x]_{3}$, one of which is diseased, three of which is disease-free, and one is uncertain. Therefore, conflicting information is common in an information system. All the condition attributes in the equivalence class $[x]_{3}$ are the same but derive different decision results. The existence of conflicting information has an enormous influence when mining the action strategy in three regions.

Taking $[x]_{8}$ as an equivalence class that needs to be moved, the decision maker hopes that the object in the diseased region moves to the disease-free region. The object in the uncertain region will be moved to the diseased or disease-free region under further medical examination.
According to equation (5), we can mine some action strategies, that is,

$$
\begin{aligned}
& r_{[x]_{8}}{ }^{\mu \rightarrow} r_{[x]_{2}}, \\
& r_{[x]_{8}}{ }^{\rightsquigarrow \rightarrow r_{[x]_{3}}},
\end{aligned}
$$

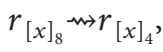

$$
\begin{aligned}
& r_{[x]_{8}}{ }^{\mu \rightarrow r_{[x]_{5}}} .
\end{aligned}
$$

The strategy $r_{[x]_{8}}{ }^{\leftrightarrow}{ }_{[x]_{3}}$ means moving objects from $[x]_{8}$ to $[x]_{3}$ according to equation (5). However, there are diseased, disease-free, and uncertain objects in $r_{[x]_{3}}$. For the 
decision maker, the outcome of movement is difficult to determine. There are different probabilities of movement:

$$
\begin{array}{r}
r_{[x]_{8}} \rightsquigarrow r_{[x]_{3}} \text { : chol: } 1 \rightsquigarrow 2 \wedge \text { bp: } 1 \rightsquigarrow 3 \wedge \text { bs: } 1 \rightsquigarrow 3 \Rightarrow \text { result }= \begin{cases}+, & =p_{1}, \\
-, & =p_{2}, \\
?, & =p_{3},\end{cases} \\
\text { subject to: age }=3 \wedge \text { sex }=0 .
\end{array}
$$

where $p_{1}, p_{2}$, and $p_{3}$ represent the proportion or probability of moving disease-free, diseased, and uncertain regions, respectively.

The existence of inconsistent information makes a lot of noisy strategies when mining action strategies, such as $r_{[x]_{8}}{ }^{\wedge} r_{[x]_{3}}$ and $r_{[x]_{8}}{ }^{\wedge} r_{[x]_{5}}$. Therefore, it is necessary to consider conflicting information and analyze strategy when mining actionable rules in three regions.

3.2. Construct Three-Way Decision Model. The actionable rules affected by inconsistent information can generate a lot of noisy strategies. These noisy strategies are not helpful to the decision maker and may cause them to make wrong judgments. Therefore, we introduce credibility and coverage concepts to construct a three-way decision model for the action strategy set.

Definition 4. (see [38]). Suppose that IS $=\{U, C \cup D, V, f\}$ is an information system, and $S$ is an action strategy mined in IS according to the target equivalence class $[x]$. The action strategy has a certain credibility. The definition is as follows:

$$
\operatorname{Credibility}(S)=\frac{\left|[x] \cap P_{j}\right|}{|[x]|},
$$

where $[x]$ is the equivalence class divided by $U /$ ind $(C), i=1,2, \ldots, m$, and $P_{j}, j=1,2,3$, is the three regions divided by evaluation function.

The strategy is certain when Credibility $(S)=1$. The strategy is uncertain when $0<\operatorname{Credibility}(S)<1$. The credibility denotes the conditional probability of target region $P_{j}$ to the target equivalence class $[x]$. When the credit is less than 1 , the same condition attributes are divided into different regions. So, credibility reflects the uncertainty of strategies.

The credibility of strategy $S$ only considers the proportion of the equivalence class $[x]$ in the target region. However, it is not enough to consider credibility in an inconsistent information system. It is necessary to consider the coverage of the strategy in the target region: how many objects induce the strategy.

Definition 5. (see [38]). Suppose that IS $=\{U, C \cup D, V, f\}$ is an information system, and $S$ is an action strategy mined in IS according to the target equivalence class $[x]$. The action strategy has a certain coverage. The definition is as follows:

$$
\operatorname{Coverage}(S)=\frac{\left|[x] \cap P_{j}\right|}{\left|P_{j}\right|},
$$

where $[x]$ is the equivalence class divided by $U /$ ind $(C), i=1,2, \ldots, m$, and $P_{j}, j=1,2,3$, is the three regions divided by evaluation function.

The coverage describes the proportion of objects meeting the strategy constraints in the target region. If the strategy's coverage is small, the objects that derive the strategy occupy a small part of the region $P_{j}$. Therefore, the causality of this strategy lacks sufficient data to support it.

Therefore, it is necessary to consider both the strategy's credibility and coverage when selecting a strategy. Based on the above description, combined with the trilevel thinking proposed by Yao [52], we constructed a novel three-way decision model for the strategy set. The model is given in Figure 2.

Given a strategy set AS $=\left\{S_{1}, S_{2}, \ldots, S_{n}\right\}$. The strategy set is divided into a top-down trilevel granularity structure according to the credibility threshold cre and the coverage threshold cov. The strategy set is gradual granulation from top to down.

The first level:

$$
\text { AS }=\left\{S_{1}, S_{2}, \ldots, S_{n}\right\} .
$$

The second level:

$$
\mathrm{AS} / \mathrm{cre}=\left\{\left\{S_{1}, S_{2}, \ldots, S_{m}\right\},\left\{S_{m+1}, S_{m+2}, \ldots, S_{n}\right\}\right\} .
$$

The third level:

$$
\mathrm{AS} / \mathrm{cre} \wedge \operatorname{cov}=\left\{\left\{S_{1}, \ldots, S_{m_{1}}\right\},\left\{S_{m_{1}+1}, \ldots, S_{m}\right\}\right\} \cup\left\{\left\{S_{m+1}, \ldots, S_{n_{1}}\right\},\left\{S_{n_{1}+1}, \ldots, S_{n}\right\}\right\} .
$$




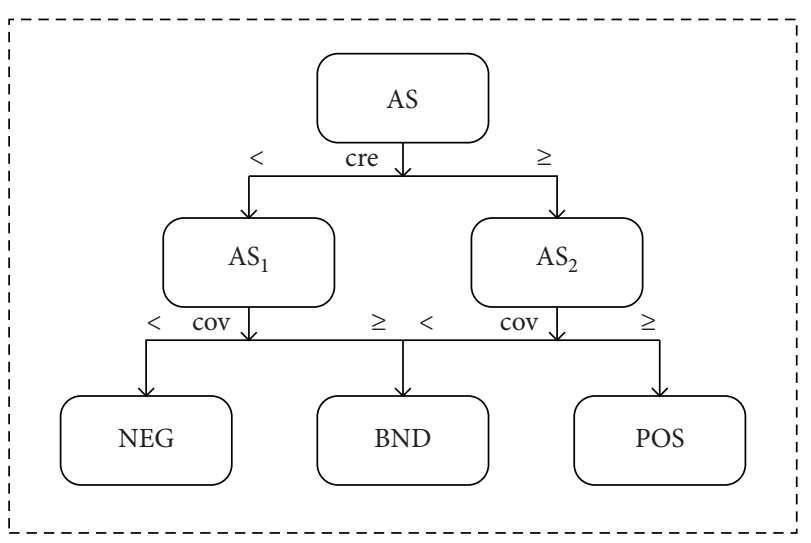

FIgURE 2: Three-way decision model for the strategy set.

The model of three-way decision for strategy set is as follows:

$$
\begin{aligned}
\operatorname{POS}(S) & =\left\{S_{i} \in S \mid \operatorname{credibility}\left(S_{i}\right) \geq \operatorname{cre} \wedge \text { coverage }\left(S_{i}\right) \geq \operatorname{cov}\right\} \\
\operatorname{NEG}(S) & =\left\{S_{i} \in S \mid \operatorname{credibility}\left(S_{i}\right) \leq \operatorname{cre} \wedge \text { coverage }\left(S_{i}\right) \leq \operatorname{cov}\right\} \\
\operatorname{BND}(S) & =(\operatorname{POS}(S) \cup \operatorname{NEG}(S))^{C} \\
& =\left\{S_{i} \in S \mid \operatorname{credibility}\left(S_{i}\right)>\operatorname{acc} \wedge \operatorname{coverage}\left(S_{i}\right)<\operatorname{cov}\right\} \cup \\
& \mid\left\{S_{i} \in S \mid \operatorname{credibility}\left(S_{i}\right)<\operatorname{cre} \wedge \operatorname{coverage}\left(S_{i}\right)>\operatorname{cov}\right\}
\end{aligned}
$$

The model introduces the concepts of credibility and coverage. In the first level, the strategy set does not introduce any concept. In the second level, the strategy set is divided into two subsets $\mathrm{AS}_{1}$ and $\mathrm{AS}_{2}$ through the credibility threshold cre. In the third level, the subsets $\mathrm{AS}_{1}$ and $\mathrm{AS}_{2}$ are classified again by the coverage threshold cov, which are above the threshold cov and below the threshold cov. Finally, the strategies whose credibility and coverage are higher than the threshold cre and cov are divided into POS region; the strategies whose accuracy and coverage are lower than the threshold cre and cov are divided into NEG region, and the remaining strategies are divided into $\mathrm{BND}$ region. The decision maker will give priority to the strategy in the POS region when selecting a strategy. The model enables the decision maker to choose a strategy with high credibility and coverage as much as possible, which can effectively avoid the influence of conflicting information.

At last, we summarize the key steps to construct the three-way decision for the strategy set. The approach is outlined in Algorithm 1.

\section{Optimal Action Strategy Selection in M-3WD}

This section analyzed the probabilistic preference in three regions and proposed an evidence theory-based approach to determine the probability of movement. The determination of the movement's probability is the basis for selecting the optimal action strategy. The optimal strategy is selected by comparing the difference between the ideal movement and the actual movement.
4.1. Probabilistic Preference in M-3WD. The model of the three-way decision for the strategy set can effectively avoid the influence of conflicting information by mining action strategy with high credibility and coverage. However, there may be many action strategies with high credibility and coverage. Therefore, we need to determine the probability of moving to three regions under different action strategies, which will help us select an optimal action strategy.

In a movement-based three-way decision, the decision maker has different preferences for different regions. For example, doctors want patients to move from a diseased region to a disease-free region in medical decision-making. According to the preference of decision makers, there are two types of movement. If the object's movement matches the preference of decision makers, we call it a favorable movement. If the object's movement does not match decision makers' preference, we call it an unfavorable movement. In a movement-based three-way decision, we first divide a set of objects into three different regions according to decision makers' needs. Then, we can mine action strategies between different regions.

Definition 6. Suppose that $\pi=\left\{P_{1}, P_{2}, P_{3}\right\}$ is a tripartition regions. We can mine action strategies and construct corresponding strategy sets:

$$
\text { AS }=\left(S_{1}, S_{2}, \ldots, S_{n}\right)
$$

where AS denotes the set of all strategies for objects in $P_{i}$ region to move to $P_{j}$ region. Objects in three regions may have nine movements:

$$
\begin{aligned}
& P_{1} \rightsquigarrow P_{1}^{\prime}, P_{1} \rightsquigarrow P_{2}^{\prime}, P_{1} \rightsquigarrow P_{3}^{\prime}, \\
& P_{2} \rightsquigarrow P_{1}^{\prime}, P_{2} \rightsquigarrow P_{2}^{\prime}, P_{2} \rightsquigarrow P_{3}^{\prime}, \\
& P_{3} \rightsquigarrow P_{1}^{\prime}, P_{3} \rightsquigarrow P_{2}^{\prime}, P_{3} \rightsquigarrow P_{3}^{\prime},
\end{aligned}
$$

where $P_{i} \rightsquigarrow P_{j}^{\prime}, i, j=1,2,3$ denotes the object in $P_{i}$ region move to $P_{j}$ region and $P_{i}$ and $P_{i}^{\prime}$ denote the region before and after the movement. In movement-based three-way decision, each kind of movement will generate the corresponding strategy set AS.

Due to the inconsistent information in the information system, the object moves from the unfavorable region to the favorable region with probability. According to the preference of decision maker, the probabilistic preference [53] movement matrix can be constructed as follows:

$$
\begin{aligned}
& p_{11}, p_{12}, p_{13}, \\
& p_{21}, p_{22}, p_{23}, \\
& p_{31}, p_{32}, p_{33},
\end{aligned}
$$

where $p_{i j}$ denotes the probability of object's movement from $P_{i}$ region to $P_{j}$ region.

How to determine the probability of movement is an urgent problem to be solved. Considering the advantages of evidence theory in information fusion, we use the credibility of the equivalence class divided under a particular flexible attribute as the mass function of evidence theory. The 
Input: IDIS $=(U, C \cup D, V, f)$, evaluation function $E(x)$, a pair of thresholds $(\alpha, \beta)$, cre, and cov.

Output: The three-way decision for strategy set.

(1) Divide equivalence classes according to condition attributes $C$.

(2) for $x \in U$ do

(3) Calculate the evaluation function $E(x)$.

(4) if $E(x) \geq \alpha$ then

(5) Divide $x$ into $P_{1}$ region.

(6) end

(7) else if $E(x) \leq \beta$ then

(8) Divide $x$ into $P_{3}$ region.

(9) end

(10) else

(11) Divide $x$ into $P_{2}$ region.

(12) end

(13) Choose equivalence classes $[x]$ that need to be moved.

(14) Mining action strategies in $P_{1}, P_{2}$, and $P_{3}$ to generate corresponding strategy set AS.

(15) end

(16) for $S \in$ AS do

(17) if credibility $(S) \geq \operatorname{cre} \wedge$ coverage $(S) \geq \operatorname{cov}$ then

(18) Divide $S$ into POS region.

(19) end

(20) else if credibility $(S)<\operatorname{cre} \wedge$ coverage $(S)<\operatorname{cov}$ then

(21) Divide $S$ into NEG region.

(22) end

(23) else

(24) Divide $S$ into BND region.

(25) end

(26) end

Algorithm 1: Three-way decision for the action strategy set.

probability of movement can be obtained by fusing multiple mass function under flexible attributes.

Evidence theory, also known as D-S evidence theory, is an uncertainty reasoning theory first proposed by Dempster. Shafer further researched and developed it. Evidence theory has been widely used in many fields such as information fusion [54], medical diagnosis [55], and uncertainty decision-making [56]. Suppose that $m: 2^{\Theta} \longrightarrow[0,1]$ is a function on the recognition framework $\Theta$. The function satisfies the following two conditions:

$$
\text { (2) } \begin{aligned}
(1) m(\phi) & =0, \\
\sum_{A \subseteq \Theta} m(A) & =1,
\end{aligned}
$$

where the subset $A$ denotes the focal elements, which meet the $m(A)>0$. The value of $m(A)$ denotes the belief that supports the occurrence of $A$. The function is called a mass function or a basic credibility distribution function.

The synthesis rule [57] is the core of evidence theory. If there is multiple evidence under the recognition framework, different mass functions will be derived. The evidence synthesis formula synthesizes multiple mass functions to obtain a fused mass function. The fused mass function is used for decision-making, which can improve accuracy. Given the recognition framework $\Theta$, for $\forall A \subseteq \Theta$, two mass functions under the same recognition frame $\Theta$ obtained from different evidences are $m_{1}$ and $m_{2}$. The synthesis formula of evidence $m_{1}$ and $m_{2}$ is as follows:

$$
\left(m_{1} \oplus m_{2}\right)(A)=\frac{1}{1-K} \sum_{B \cap C=A} m_{1}(B) m_{2}(C), K=\sum_{B \cap C=\phi} m_{1}(B) m_{2}(C)
$$

where $K$ is the confidence assigned to the null value. In reality, the null value has no confidence, that is, $m(\phi)=0$. When the two pieces of evidence are combined, the constraint condition $K<1$ must be satisfied. If $K=1$, the evidence is completely contradictory, and there is no $m_{1} \oplus m_{2}$ at this time.
We use the credibility cre $_{a j}$ of the equivalence class $[x]$ under a certain flexible attribute $a \in C$ as the mass function, that is, $m\left(P_{j} /[x]_{a}\right)$. The meaning of credibility is the proportion of equivalence classes divided by the condition attribute $a \in C$ in the target region $P_{j}$. The larger the ratio, the higher the 
reliability. The mass function needs to satisfy two constraints in equation (20). The corresponding proof is as follows.

Proof. The mass function needs to satisfy two constraints, namely, $m(\phi)=0$ and $\sum_{A \in \Theta} m(A)=1$. According to the definition of credibility,

$$
\begin{aligned}
m\left(\phi /[x]_{a}\right) & =\frac{\left|[x]_{a} \cap \phi\right|}{\left|[x]_{a}\right|}=0, \\
\sum_{j=1}^{3} m\left(\frac{P_{j}}{[x]_{a}}\right) & =\sum_{j=1}^{3} \frac{\left|[x]_{a} \cap P_{j}\right|}{\left|[x]_{a}\right|}=\frac{\left|[x]_{a} \cap\left(P_{1} \cup P_{2} \cup P_{3}\right)\right|}{\left|[x]_{a}\right|}=1 .
\end{aligned}
$$

The credibility satisfies the above two constraints.

The credibility uses the value of each attribute and the decision result, that is, it makes full use of all the information in the information system. Therefore, the movement probability determined by evidence theory is more accurate.

The importance of different attributes to the decision maker is different. Considering the need for accuracy, the decision maker needs to consider the importance of different attributes when synthesizing the approximate movement probability. The attribute importance is taken as the weight factor, the greater the attribute's importance, the greater the weight of the mass function. The weight of the mass function under different attributes is represented by $w_{a}$.

Suppose that IS $=(U, C \cup D, V, f)$ is an information system, action strategies $S$ derived in IS, the weight $w_{a}$ of attribute $a$, and the mass function $m\left(P_{j} /[x]_{a}\right)$. The model of approximate movement probability assignment in three regions is as follows:

$$
m\left(P_{j}\right)= \begin{cases}0, & \frac{P_{j}}{[x]}=\phi, \\ \frac{\sum_{\cap P_{j} /[x]_{a}=P_{j}} \prod_{a \in C} w_{a} m\left(P_{j} /[x]_{a}\right)}{1-\sum_{1 \leq i \leq m} \prod_{a \in C} w_{a} m\left(P_{j} /[x]_{a}\right)}, & \frac{P_{j}}{[x]} \neq \phi . \\ \cap P_{j} /[x]_{a}=\phi & \end{cases}
$$

The preferences of movement widely exist in the movement-based three-way decision. The preferences are often expressed in the form of probability. We analyze the probabilistic preference in the movement-based three-way decision. The evidence theory-based method is proposed to determine the probability of movement. The model uses all the information in the system and considers the importance of different attributes to improve the fusion results' reliability and objectivity.

4.2. Optimal Action Strategy Selection. The movement-based three-way decision model aims to move objects from the unfavorable region to the favorable region. The movement of objects brings benefits and costs. We can select the optimal action strategy by analyzing the benefits and costs. However, the benefits and costs are difficult to determine. To select an optimal action strategy, we propose an action strategy selection method by analyzing the difference between the ideal movement and actual movement, the smaller the difference, the better the strategy. The ideal movement is determined by the decision maker, which can bring the highest utility.

Given the equivalence class $[x]=\left\{x_{1}, x_{2}, \ldots, x_{n}\right\}$ in the unfavorable region, the decision maker wants to move $[x]$ to a favorable region. We describe the equivalence class's probabilistic preference $[x]$ moving to three regions under the action strategy through a probability mass function. The probability mass function is the probability of a discrete random variable at each particular value. All values of the probability mass functions are nonnegative, and the sum of the probability is equal to 1 .

For the equivalence class $[x]$, the probability mass function of moving to three regions under the action strategy is represented by

$$
p_{r}=\left\{p\left(x_{1}\right), p\left(x_{2}\right), \ldots, p\left(x_{n}\right)\right\} \text {. }
$$

For example, objects in the equivalence class $[x]=\left\{x_{1}, x_{2}, x_{3}, x_{4}, x_{5}\right\}$ are in $P_{3}$ region. After the action strategy, the distribution of objects in three regions is as follows:

$$
\begin{array}{r}
P_{1}=\left\{x_{1}, x_{3}, x_{4}\right\}, \\
P_{2}=\left\{x_{2}\right\}, \\
P_{3}=\left\{x_{5}\right\} .
\end{array}
$$

The probability mass function of $[x]$ under the action strategy is $p_{r}=\{0.6,0.2,0.6,0.6,0.2\}$. It can be seen from the probability mass function that $p\left(P_{1}\right)+p\left(P_{2}\right)+p\left(P_{3}\right)$ $=0.6+0.2+0.2=1$. The probability mass function of ideal movement for decision maker is as follows:

$$
p_{e}=\left\{p\left(x_{1}\right), p\left(x_{2}\right), \ldots, p\left(x_{n}\right)\right\} .
$$

We use $u_{p_{j}}(x), j=1,2,3$, to represent the quality or utility of object $x$ in different regions. So, the quality or utility of $[x]$ in three regions is represented as follows: 


$$
U([x])=\left\{u_{P_{j}}\left(x_{1}\right), u_{P_{j}}\left(x_{2}\right), \ldots, u_{P_{j}}\left(x_{n}\right)\right\} .
$$

Often objects have different utilities in different regions and have the same utility in the same region. For example, in an opinion poll, we usually divide voters into three types, those who support the candidate, those who oppose the candidate, and those who are neutral. The utility of the same type of voters is the same, and the utility of different types of voters is different. The utility of voters who support the candidate is more than the voters who neutral. The utility of voters who are neutral is more than voters who oppose the candidate.

The moment is the statistics commonly used. Moments of each order are often used to describe distribution characteristics. Moments have been fully applied in areas such as risk analysis and insurance dividends [58]. Suppose that $X$ is a discrete random variable. We get the probability of all possible value of $x$, that is,

$$
\begin{aligned}
P\left(X=x_{k}\right) & =p_{k}, \\
k & =1,2, \ldots, n .
\end{aligned}
$$

The mathematical expectation of $X$ is expressed as $E(X)$ and the $k$-order moment of $X$ is expressed as $E\left(X^{k}\right)(k=1,2, \ldots, n)$. The first moment denotes the expectation and the second moment denotes the variance.

Given a discrete random variable $X$ and its probability mass function $p(x)$, the moment generating function of [59] $X$ is expressed as $M_{X}(t)$. The power series expansion of the function is as follows:

$$
M_{X}(t)=\sum_{k=1}^{\infty} \frac{E\left(X^{k}\right)}{k !} t^{k}, \quad t \in R,
$$

where $E(X)$ represents the expectation of the random variable $X$ and $E\left(X^{2}\right)$ represents the variance of the random variable $X$. The moment generating function is a real-valued function of a random variable.

The optimal strategy not only has the highest expectations but also has the least uncertainty. Therefore, we use the first two order moments to construct the degree of difference between the ideal movement and the actual movement. The formula is as follows:

$$
\begin{aligned}
D\left(p_{r} \| p_{e}, S\right) & =\left\{\sum_{k=1}^{2} \frac{\left[E\left(p_{e}^{k}\right)-E\left(p_{r}^{k}\right)\right]^{2}}{k !}\right\}^{1 / 2}, \\
E\left(X^{1}\right) & =\sum_{k=1}^{n} u_{P_{j}}\left(x_{k}\right) p\left(x_{k}\right), E\left(X^{2}\right)=\frac{\sum_{k=1}^{n}\left(u_{P_{j}}\left(x_{k}\right)-E\left(X^{1}\right)\right)^{2}}{n},
\end{aligned}
$$

where the first-order moment is the expectation and the second-order moment is the variance.

The proposed method selects an optimal action strategy by comparing the ideal movement and the actual movement, the smaller the difference, the better the strategy. The first moment compares the expectations between the ideal movement and the actual movement. The second-order moment compares the variance between the ideal movement and the actual movement. The framework can further consider the variance based on the expectations and help the decision maker select an optimal action strategy.

Finally, we summarize the key steps of approximate movement probability assignment and effectiveness measures framework for the trisecting-acting-outcome model. The specific procedure is given in Algorithm 2.

\section{An Illustrative Example}

In this section, we use an example of medical decision in Table 2 to illustrate the proposed method. There are 468 suspected patients and six symptoms or attributes. Chol, bp, and bs stand for cholesterol level, blood pressure, and blood sugar, respectively. The condition attributes are age, sex, chol, bp, and bs. The result is a decision attribute. Symbols "+," "-," and "?" stand for a suspected patient who has the disease, does not have the disease, and uncertain, respectively. For the convenience of representation, we divide objects into ten equivalence classes based on condition attributes. The right side of the decision attribute value indicates the number of objects in the equivalence class whose decision result is this type. For example, there are 50 objects in $[x]_{1}$, of which there are five objects with a decision result of "+," 30 objects with a decision result of " - ," and 15 objects with a decision result of "?." We divide all objects into $P_{1}, P_{2}$, and $P_{3}$ according to the value "-," "+," and "?" of the decision attribute.

Taking $[x]_{10}$ as an example of the equivalence class that needs to be moved, from the information table, there are 105 objects in the equivalence class. Among them, 0 object does not have the disease, 100 objects have the disease, and 5 objects are uncertain. For diseased objects in $[x]_{10}$, action strategy are mined from the table according to equations (4) 
Input: IDIS $=(U, C \cup D, V, f), P_{1}, P_{2}, P_{3}$, and strategy set AS.

Output: effectiveness of strategy $S$.

(1) for $a \in A_{f}$ do

(2) Divide equivalence classes $[x]_{a}$.

(3) Calculate credibility cre $_{a}$ under flexible attribute a.

(4) Assign weight for attribute $a$

(5) end

(6) Synthesize movement probability according to model of approximate movement probability assignment model $m\left(P_{j}\right)$.

(7) min difference $=\infty$.

(8) for $S \in$ AS do

(9) Formulate the probability quality function pe ideally expected by decision maker.

(10) Calculate the probability quality function pr according to model of approximate movement probability assignment.

(11) Calculate the degree of difference by $D\left(p_{r} \| p_{e}, S\right)$

(12) if $D\left(p_{r} \| p_{e}, S\right) \leq$ mindifference then

(13) $\quad$ min difference $=D\left(p_{r} \| p_{e}, S\right) \leq$

(14) end

(15) end

Algorithm 2: The algorithm for selecting an optimal action strategy.

TAble 2: Table for medical decision-making.

\begin{tabular}{lcccccccc}
\hline & Age & Sex & chol & bp & bs & & Result & \\
\hline$[x]_{1}$ & 3 & 1 & 2 & 1 & 1 & $+=5$ & $-=30$ & $?=15$ \\
{$[x]_{2}$} & 3 & 0 & 2 & 2 & 2 & $+=40$ & $-=5$ & $?=5$ \\
{$[x]_{3}$} & 2 & 1 & 3 & 2 & 3 & $+=30$ & $-=4$ & $?=6$ \\
{$[x]_{4}$} & 3 & 0 & 2 & 3 & 2 & $+=35$ & $-=10$ & $?=5$ \\
{$[x]_{5}$} & 3 & 0 & 1 & 2 & 1 & $+=15$ & $-=15$ & $?=20$ \\
{$[x]_{6}$} & 1 & 1 & 3 & 2 & 2 & $+=30$ & $-=6$ & $?=4$ \\
{$[x]_{7}$} & 3 & 0 & 1 & 1 & 2 & $+=2$ & $-=0$ & $?=0$ \\
{$[x]_{8}$} & 3 & 0 & 1 & 2 & 3 & $+=20$ & $-=10$ & $?=10$ \\
{$[x]_{9}$} & 2 & 0 & 1 & 2 & 2 & $+=30$ & $-=5$ & $?=6$ \\
{$[x]_{10}$} & 3 & 0 & 3 & 3 & 3 & $+=0$ & $-=100$ & $?=5$ \\
\hline
\end{tabular}

and (5), where age and sex are stable attributes and chol, bp, and bs are flexible attributes. The action strategy of $[x]_{10}$ is as follows:

$$
\begin{aligned}
& S_{1}=\text { chol: } 3 \rightsquigarrow 2 \wedge \text { bp: } 3 \rightsquigarrow 2 \wedge \text { bs: } 3 \rightsquigarrow 2, \\
& S_{2}=\text { chol: } 3 \rightsquigarrow 2 \wedge \text { bp: } 3 \rightsquigarrow 3 \wedge \text { bs: } 3 \rightsquigarrow 2, \\
& S_{3}=\text { chol: } 3 \rightsquigarrow 2 \wedge \text { bp: } 3 \rightsquigarrow 2 \wedge \text { bs: } 3 \rightsquigarrow 1, \\
& S_{4}=\text { chol: } 3 \rightsquigarrow 1 \wedge \text { bp: } 3 \rightsquigarrow 1 \wedge \text { bs: } 3 \rightsquigarrow 2, \\
& S_{5}=\text { chol: } 3 \rightsquigarrow 1 \wedge \text { bp: } 3 \rightsquigarrow 2 \wedge \text { bs: } 3 \rightsquigarrow 3 .
\end{aligned}
$$

Mining actionable rules in three regions and generating the corresponding strategy set $S=\left\{S_{1}, S_{2}, S_{3}, S_{4}, S_{5}\right\}$, calculate the credibility and coverage of each strategy in the strategy set, and the result is shown in Table 3.

Setting the credibility threshold to 0.5 and the coverage threshold to 0.1 to construct a three-way decision model for the strategy set, the model divides the strategy set into three regions POS, BND, and NEG through thresholds of accuracy and coverage. Each subset contains the following strategies:

$$
\begin{aligned}
\mathrm{POS} & =\left\{S_{1}, S_{2}\right\}, \\
\mathrm{BND} & =\left\{S_{4}, S_{5}\right\}, \\
\mathrm{NEG} & =\left\{S_{3}\right\} .
\end{aligned}
$$

TABLE 3: Credibility and coverage of each strategy.

\begin{tabular}{lccccc}
\hline & $S_{1}$ & $S_{2}$ & $S_{3}$ & $S_{4}$ & $S_{5}$ \\
\hline cre & $40 / 50$ & $35 / 50$ & $15 / 50$ & $2 / 2$ & $20 / 40$ \\
cov & $40 / 207$ & $35 / 207$ & $15 / 207$ & $2 / 207$ & $20 / 207$ \\
\hline
\end{tabular}

The three regions have a partial order relationship of POS $>$ BND $>$ NEG. When the decision maker chooses a strategy, the strategy in the POS region is preferred. The accuracy of strategy $S_{4}$ is 1 , but the coverage is $2 / 207$. Two hundred and seven objects have no disease in the information system, but only two objects derived strategy $S_{4}$. This strategy is most likely a noise strategy that is affected by inconsistent information. This strategy is not helpful for the decision maker. Therefore, the strategy $S_{4}$ is effectively divided into the BND region. The strategy $S_{1}$ has an credibility of $40 / 50$ and coverage of 40/207. Both credibility and coverage exceed the thresholds. Therefore, strategy $S_{1}$ is more effective and more reliable.

Both credibility and coverage are positive evaluation criteria, that is, the higher the value of two criteria, the better the strategy. This model can effectively avoid the influence of conflicting information for the decision maker. There are two action strategies in the POS region: $S_{1}$ and $S_{2}$. They both have high credibility and coverage. We select an optimal action strategy by the approximate movement probability assignment model and the effectiveness measures the framework.

The credibility of all flexible attributes of the strategies $S_{1}$ and $S_{2}$ in the POS region is used as a mass function. The approximate movement probability assignment is synthesized by the mass function under each flexible attributes. A total of 150 decision objects derived from strategy $S_{1}$ under the attribute chol, 80 of them do not have the disease, 45 of them have the disease, and 25 of them are uncertain. Strategy $S_{1}$ has a total of 261 objects under the attribute bp, 165 of them have not the disease, 45 of them have the disease, and 51 of them are uncertain. The strategy $S_{1}$ has a total of 183 
objects under the attribute bs, 137 of them do not have the disease, 26 of them have the disease, and 20 are uncertain. The basic credibility allocation of strategy under the three attributes is shown in Table 4 .

Given the flexible attribute chol, bp, and bs. The attribute weights are as follows:

$$
\begin{gathered}
w_{\text {chol }}=\frac{1}{3}, \\
w_{\text {bp }}=\frac{1}{3}, \\
w_{\text {bs }}=\frac{1}{3} .
\end{gathered}
$$

The approximate movement probability distribution of strategy $S_{1}$ is calculated by equation (23):

$$
\begin{gathered}
m\left(P_{1}\right)=\frac{\sum_{\cap P_{1} /[x]_{a}=P_{1}} \prod_{a \in C} w_{a} m\left(P_{1} /[x]_{a}\right)}{\prod_{a \in C} w_{a} m\left(P_{1} /[x]_{a}\right)}=0.96, \\
\sum_{1 \leq i \leq m} P_{1} /[x]_{a}=\phi \\
m\left(\left(P_{2}\right)=\frac{\sum_{\cap P_{2} /[x]_{a}=P_{2}} \prod_{a \in C} w_{a} m\left(P_{2} /[x]_{a}\right)}{1-\sum_{1 \leq i \leq m} \prod_{a \in C} w_{a} m\left(P_{2} /[x]_{a}\right)}=0.03,\right. \\
\cap P_{2} /[x]_{a}=\phi \\
m\left(\left(P_{3}\right)=\frac{\sum_{\cap P_{3} /[x]_{a}=P_{3}} \prod_{a \in C} w_{a} m\left(P_{3} /[x]_{a}\right)}{1-\sum_{1 \leq i \leq m} \prod_{a \in C} w_{a} m\left(P_{3} /[x]_{a}\right)}=0.01 .\right. \\
\cap P_{3} /[x]_{a}=\phi
\end{gathered}
$$

The basic credibility allocation of strategy $S_{2}$ under the three attributes is shown in Table 5. The approximate movement probability allocation of strategy $S_{2}$ is calculated by equation (23):

$$
\begin{aligned}
& m\left(P_{1}\right)=\frac{\sum_{\cap P_{1} /[x]_{a}=P_{1}} \prod_{a \in C} w_{a} m\left(P_{1} /[x]_{a}\right)}{1-\sum_{1 \leq i \leq m} \quad \prod_{a \in C} w_{a} m\left(P_{1} /[x]_{a}\right)}=0.74, \\
& \cap P_{1} /[x]_{a}=\phi \\
& m\left(P_{2}\right)=\frac{\sum_{\cap P_{2} /[x]_{a}=P_{2}} \prod_{a \in C} w_{a} m\left(P_{2} /[x]_{a}\right)}{1-\sum_{1 \leq i \leq m} \quad \prod_{a \in C} w_{a} m\left(P_{2} /[x]_{a}\right)}=0.25 \\
& \cap P_{2} /[x]_{a}=\phi
\end{aligned}
$$

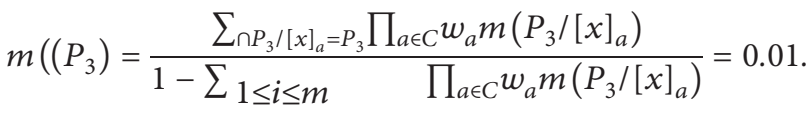

$$
\begin{aligned}
& \cap P_{3} /[x]_{a}=\phi
\end{aligned}
$$

TABLE 4: Basic credibility assignment of strategy $S_{1}$.

\begin{tabular}{lccc}
\hline & $P_{1}$ & $P_{2}$ & $P_{3}$ \\
\hline$m$ (chol) & $80 / 150$ & $45 / 150$ & $25 / 150$ \\
$m$ (bp) & $165 / 261$ & $45 / 261$ & $51 / 261$ \\
$m$ (bs) & $137 / 183$ & $26 / 183$ & $20 / 183$ \\
\hline
\end{tabular}

The probability quality function that the decision-maker ideal movement is pe and the probability quality of strategies $S_{1}$ and $S_{2}$ is $\operatorname{pr}_{S_{1}}$ and $\operatorname{pr}_{S_{2}}$, respectively. Their utility is expressed as follows:

$$
\begin{gathered}
U_{\mathrm{pe}}\left([x]_{10}\right)=\left\{\left\{u_{P_{1}}(x)\right\} \times 100,\left\{u_{P_{2}}(x)\right\} \times 0,\left\{u_{P_{3}}(x) \times 0\right\}\right\}, \\
U_{\mathrm{pr}_{S_{1}}}\left([x]_{10}\right)=\left\{\left\{u_{P_{1}}(x)\right\} \times 96,\left\{u_{P_{2}}(x)\right\} \times 3,\left\{u_{P_{3}}(x) \times 1\right\}\right\}, \\
U_{\mathrm{pr}_{S_{2}}}\left([x]_{10}\right)=\left\{\left\{u_{P_{1}}(x)\right\} \times 74,\left\{u_{P_{2}}(x)\right\} 25,\left\{u_{P_{3}}(x) \times 1\right\}\right\} .
\end{gathered}
$$

In medical decision-making, the decision maker wants all objects in the diseased region to move to the disease-free region with appropriate action strategies, which will bring the greatest benefits to the decision-maker. The decision maker wants that the object in the diseased region in $[x]_{10}$ to move completely to the disease-free region with appropriate strategies. Therefore, the ideal probability distribution of the decision maker is recorded as $p_{e}=[1,0,0]$. Due to the influence of random errors in the actual cases, the strategy's effect is often difficult to meet the expectations of the decision maker. The above approximate movement probability allocation is used as its real movement probability distribution.

The objects in the $P_{1}, P_{2}$, and $P_{3}$ regions are assigned utility of 3,1 , and 2 , respectively. That is to say, the utility of the object in the not disease region is 3 , the utility of the object in the diseased region is 1 , and the utility of the object in the uncertainty area is 2 . According to equation (30), we can calculate the difference between the ideal expectation of the decision maker and strategy $S_{1}$ :

$$
\left.D\left(p_{r} \| p_{e}\right), S_{1}\right)=\left\{\sum_{k=1}^{2} \frac{\left[E\left(p_{e}^{k}\right)-E\left(p_{r}^{k}\right)\right]^{2}}{k !}\right\}^{1 / 2}=0.1128
$$

The difference between the ideal expectation of the decision maker and strategy $S_{2}$ is as follows:

$$
\left.D\left(p_{r} \| p_{e}\right), S_{2}\right)=\left\{\sum_{k=1}^{2} \frac{\left[E\left(p_{e}^{k}\right)-E\left(p_{r}^{k}\right)\right]^{2}}{k !}\right\}^{1 / 2}=0.7357
$$

The degree of difference of $S_{1}$ is smaller, that is to say, the action strategy $S_{1}$ is more effective, with less uncertainty and more in line with the needs of the decision maker. 
TABLe 5: Basic credibility allocation of strategy $S_{2}$.

\begin{tabular}{lccc}
\hline & $P_{1}$ & $P_{2}$ & $P_{3}$ \\
\hline$m$ (chol) & $80 / 150$ & $45 / 150$ & $25 / 150$ \\
$m$ (bp) & $35 / 155$ & $110 / 155$ & $10 / 551$ \\
$m$ (bs) & $137 / 183$ & $26 / 183$ & $20 / 183$ \\
\hline
\end{tabular}

\section{Conclusion}

In this paper, we analyze the existence of conflicting information in a movement-based three-way decision. We construct a three-way decision model for the action strategy set by introducing the credibility and coverage. The model can effectively avoid the influence of conflicting information. To select an optimal action strategy, we analyzed the probabilistic preference in a movement-based three-way decision. An evidence-theory based method for determining the probability of movement has been proposed. The optimal action strategy can be selected by analyzing the difference between the ideal movement and the actual movement, the smaller the difference, the better the strategy. We illustrate the practicability of the proposed method through an example of medical decision-making. This model improves and enriches the movement-based three-way decision and widens its application range.

In future work, we will systematically study conflicting information in movement-based three-way decisions and establish a more effective evaluation framework to evaluate and select the optimal action strategy. Thresholds for credibility and coverage are given by expert experience, which is too subjective. In the future, we will study how to determine optimal thresholds. This paper only considers the case where the same region's objects have the same quality or utility. Therefore, the application of utility theory is also a future research direction.

\section{Data Availability}

All data, models, and code generated or used during the study are included within the article.

\section{Conflicts of Interest}

The authors declare that they have no conflicts of interest.

\section{Acknowledgments}

This work was supported in part by the Natural Science Foundation of Heilongjiang Province (LH2020F031).

\section{References}

[1] Y. Yao, "An outline of a theory of three-way decisions," in Proceedings of the International Conference on Rough Sets and Current Trends in Computing, pp. 1-17, Springer, Berlin, Germany, 2012.

[2] Y. Yao, "Three-way decision and granular computing," International Journal of Approximate Reasoning, vol. 103, pp. 107-123, 2018.
[3] C. Gao and Y. Yao, "Actionable strategies in three-way decisions," Knowledge-Based Systems, vol. 133, pp. 141-155, 2017.

[4] D. Liu and D. Liang, "Generalized three-way decisions and special three-way decisions," Journal of Frontiers of Computer Science and Technology, vol. 11, pp. 502-510, 2017.

[5] X. Deng and Y. Yao, "An information-theoretic interpretation of thresholds in probabilistic rough sets," in Proceedings of the International Conference on Rough Sets and Knowledge Technology, pp. 369-378, Springer, New York, NY, USA, 2012.

[6] Y. Zhang and J. Yao, "Gini objective functions for three-way classifications," International Journal of Approximate Reasoning, vol. 81, pp. 103-114, 2017.

[7] M. K. Afridi, N. Azam, and J. Yao, "Variance based three-way clustering approaches for handling overlapping clustering," International Journal of Approximate Reasoning, vol. 118, pp. 47-63, 2020.

[8] P. Wang, H. Shi, X. Yang, and J. Mi, “Three-way k-means: integrating k-means and three-way decision," International Journal of Machine Learning and Cybernetics, vol. 10, no. 10, pp. 2767-2777, 2019.

[9] H. Yu, Y. Chen, P. Lingras, and G. Wang, "A three-way cluster ensemble approach for large-scale data," International Journal of Approximate Reasoning, vol. 115, pp. 32-49, 2019.

[10] H. Yu, P. Jiao, Y. Yao, and G. Wang, "Detecting and refining overlapping regions in complex networks with three-way decisions," Information Sciences, vol. 373, pp. 21-41, 2016.

[11] H. Yu, Z. Liu, and G. Wang, "An automatic method to determine the number of clusters using decision-theoretic rough set," International Journal of Approximate Reasoning, vol. 55, no. 1, pp. 101-115, 2014.

[12] H. Yu, X. Wang, G. Wang, and X. Zeng, "An active three-way clustering method via low-rank matrices for multi-view data," Information Sciences, vol. 507, pp. 823-839, 2020.

[13] J. Qi, T. Qian, and L. Wei, "The connections between threeway and classical concept lattices," Knowledge-Based Systems, vol. 91, pp. 143-151, 2016.

[14] H. Zhi, J. Qi, T. Qian, and L. Wei, “Three-way dual concept analysis," International Journal of Approximate Reasoning, vol. 114, pp. 151-165, 2019.

[15] G. Lang, J. Luo, and Y. Yao, "Three-way conflict analysis: a unification of models based on rough sets and formal concept analysis," Knowledge-Based Systems, vol. 194, 2020.

[16] Y. Yao, "Three-way conflict analysis: reformulations and extensions of the pawlak model," Knowledge-Based Systems, vol. 180, pp. 26-37, 2019.

[17] M. K. Afridi, N. Azam, J. Yao, and E. Alanazi, "A three-way clustering approach for handling missing data using gtrs," International Journal of Approximate Reasoning, vol. 98, pp. 11-24, 2018.

[18] Y. Zhang and J. Yao, "Game theoretic approach to shadowed sets: a three-way tradeoff perspective," Information Sciences, vol. 507, pp. 540-552, 2020.

[19] J. Yao and N. Azam, "Web-based medical decision support systems for three-way medical decision making with gametheoretic rough sets," IEEE Transactions on Fuzzy Systems, vol. 23, pp. 3-15, 2014.

[20] X. Yang, T. Li, H. Fujita, D. Liu, and Y. Yao, “A unified model of sequential three-way decisions and multilevel incremental processing," Knowledge-Based Systems, vol. 134, pp. 172-188, 2017.

[21] X. Yang, T. Li, D. Liu, and H. Fujita, "A temporal-spatial composite sequential approach of three-way granular computing," Information Sciences, vol. 486, pp. 171-189, 2019. 
[22] D. Liang, M. Wang, Z. Xu, and D. Liu, "Risk appetite dual hesitant fuzzy three-way decisions with todim," Information Sciences, vol. 507, pp. 585-605, 2020.

[23] M. Wang, D. Liang, and Z. Xu, "Sequential three-way multiple attribute group decisions with individual attributes and its consensus achievement based on social influence," Information Sciences, vol. 518, pp. 286-308, 2020.

[24] Y. Yao, "Three-way decisions with probabilistic rough sets," Information Sciences, vol. 180, no. 3, pp. 341-353, 2010.

[25] J. Qi, L. Wei, and Y. Yao, "Three-way formal concept analysis," in Proceedings of the International Conference on Rough Sets and Knowledge Technology, pp. 732-741, Springer, Berlin, Germany, 2014.

[26] H. Yu, C. Zhang, and G. Wang, "A tree-based incremental overlapping clustering method using the three-way decision theory," Knowledge-Based Systems, vol. 91, pp. 189-203, 2016.

[27] P. Wang and Y. Yao, "Ce3: a three-way clustering method based on mathematical morphology," Knowledge-based Systems, vol. 155, pp. 54-65, 2018.

[28] H. Li, L. Zhang, B. Huang, and X. Zhou, "Sequential three-way decision and granulation for cost-sensitive face recognition," Knowledge-Based Systems, vol. 91, pp. 241-251, 2016.

[29] L. Zhang, H. Li, X. Zhou, and B. Huang, "Sequential three-way decision based on multi-granular autoencoder features," Information Sciences, vol. 507, pp. 630-643, 2020.

[30] B. Zhou, Y. Yao, and J. Luo, "Cost-sensitive three-way email spam filtering," Journal of Intelligent Information Systems, vol. 42, no. 1, pp. 19-45, 2014.

[31] J. Xu, D. Miao, Y. Zhang, and Z. Zhang, "A three-way decisions model with probabilistic rough sets for stream computing," International Journal of Approximate Reasoning, vol. 88, pp. 1-22, 2017.

[32] H.-R. Zhang, F. Min, and B. Shi, "Regression-based three-way recommendation," Information Sciences, vol. 378, pp. 444461, 2017.

[33] C. Jiang, Y. Duan, and J. Yao, "Resource-utilization-aware task scheduling in cloud platform using three-way clustering," Journal of Intelligent \& Fuzzy Systems, vol. 37, no. 4, pp. 5297-5305, 2019.

[34] X. Deng and Y. Yao, "Decision-theoretic three-way approximations of fuzzy sets," Information Sciences, vol. 279, pp. 702-715, 2014.

[35] C. Jiang, D. Guo, Y. Duan, and Y. Liu, "Strategy selection under entropy measures in movement-based three-way decision," International Journal of Approximate Reasoning, vol. 37, 2020.

[36] C. Jiang and Y. Yao, "Effectiveness measures in movementbased three-way decisions," Knowledge-Based Systems, vol. 160, pp. 136-143, 2018.

[37] D. Q. Miao, Y. Zhao, Y. Y. Yao, H. X. Li, and F. F. Xu, "Relative reducts in consistent and inconsistent decision tables of the pawlak rough set model," Information Sciences, vol. 179 , no. 24 , pp. 4140-4150, 2009.

[38] Z. Pawlak, "Rough sets: theoretical aspects of reasoning about data," Springer Science \& Business Media, vol. 9, 2012.

[39] Y. Yao, "Three-way decisions and cognitive computing," Cognitive Computation, vol. 8, no. 4, pp. 543-554, 2016.

[40] Y. Yao, "Set-theoretic models of three-way decision," Granular Computing, vol. 1-16, 2014.

[41] M. Hu and Y. Yao, "Structured approximations as a basis for three-way decisions in rough set theory," Knowledge-Based Systems, vol. 165, pp. 92-109, 2019.
[42] D. Liu and D. Liang, "Three-way decisions in ordered decision system," Knowledge-Based Systems, vol. 137, pp. 182-195, 2017.

[43] J. Luo, M. Hu, and K. Qin, "Three-way decision with incomplete information based on similarity and satisfiability," International Journal of Approximate Reasoning, vol. 2, 2020.

[44] M. Gao, Q. Zhang, F. Zhao, and G. Wang, "Mean-entropybased shadowed sets: a novel three-way approximation of fuzzy sets," International Journal of Approximate Reasoning, vol. 13, 2020.

[45] X. R. Zhao and Y. Yao, "Three-way fuzzy partitions defined by shadowed sets," Information Sciences, vol. 497, pp. 23-37, 2019.

[46] D. Liang and D. Liu, "Deriving three-way decisions from intuitionistic fuzzy decision-theoretic rough sets," Information Sciences, vol. 300, pp. 28-48, 2015.

[47] D. Liang, Z. Xu, and D. Liu, "Three-way decisions with intuitionistic fuzzy decision-theoretic rough sets based on point operators," Information Sciences, vol. 375, pp. 183-201, 2017.

[48] Q. Zhang, F. Zhao, J. Yang, and G. Wang, "Three-way decisions of rough vague sets from the perspective of fuzziness," Information Sciences, vol. 50, 2020.

[49] J. Yang and Y. Yao, "Semantics of soft sets and three-way decision with soft sets," Knowledge-Based Systems, vol. 194, 2020.

[50] J. Kacprzyk, S. Zadrożny, and Z. W. Raś, "How to support consensus reaching using action rules: a novel approach," International Journal of Uncertainty, Fuzziness and Knowledge-Based Systems, vol. 18, no. 04, pp. 451-470, 2010.

[51] Z. W. Raś and A. Dardzińska, "From data to classification rules and actions," International Journal of Intelligent Systems, vol. 26, pp. 572-590, 2011.

[52] Y. Yao, "Tri-level thinking: models of three-way decision," International Journal of Machine Learning and Cybernetics, vol. 11, pp. 1-13, 2019.

[53] Y. Zhang, Z. Xu, H. Wang, and H. Liao, "Consistency-based risk assessment with probabilistic linguistic preference relation," Applied Soft Computing, vol. 49, pp. 817-833, 2016.

[54] J. Liu, X. Liao, and J.-B. Yang, "A group decision-making approach based on evidential reasoning for multiple criteria sorting problem with uncertainty," European Journal of Operational Research, vol. 246, no. 3, pp. 858-873, 2015.

[55] Y. Wang, Y. Dai, Y.-W. Chen, and F. Meng, "The evidential reasoning approach to medical diagnosis using intuitionistic fuzzy dempster-shafer theory," International Journal of Computational Intelligence Systems, vol. 8, no. 1, pp. 75-94, 2015.

[56] J. B. Yang and D. L. Xu, "On the evidential reasoning algorithm for multiple attribute decision analysis under uncertainty," IEEE Transactions on Systems, Man, and CyberneticsPart A: Systems and Humans, vol. 32, pp. 289-304, 2002.

[57] L. Han and L. P. Shi, "Approach to evidence combination based on rough set," in Proceedings of the 2009 International Conference on Electronic Computer Technology, pp. 693-697, IEEE, New York, NY, USA, 2009.

[58] Y. Huang and W. Yu, "Studies on a double Poisson-geometric insurance risk model with interference," Discrete Dynamics in Nature and Society, vol. 2013, 2013.

[59] G. Tao, L. Han, and J. Song, "Divergence measure between the probability distributions based on moments," Journal of Systems Science and Mathematical Sciences, vol. 33, pp. 1071-1082, 2013. 